\title{
Heredabilidades para características de crecimiento a través de los años en la raza Blanco Orejinegro
}

\author{
Heritability for growth characteristics through the years in the Blanco \\ Orejinegro breed
}

\author{
Juan Carlos Fernández N. ${ }^{1}$, Natalia Herrera P. ${ }^{13}$, Juan Pérez G. ${ }^{1}$, Matiluz Doria R. ${ }^{1}$, \\ Lorena Mestra V. ${ }^{1}$, Carlos Lucero C. ${ }^{2}$
}

\section{RESUMEN}

\begin{abstract}
El objetivo del estudio fue estimar los valores de heredabilidad directa, materna y las varianzas debidas al ambiente permanente desde año 1990 hasta 2012 en la raza criolla colombiana Blanco Orejinegro. Se analizaron 10698 datos sobre las variables de crecimiento para peso al nacimiento (PN), peso ajustado a los 240 días (PAJ240) y peso ajustado a los 480 días (PAJ480). Se utilizó la metodología de máxima verosimilitud restringida libre de derivadas (DFREML), ajustando un modelo animal que incluyó efectos genéticos directos, maternos y variaciones debidas al ambiente permanente, asumiendo como efectos fijos el año, época de nacimiento, el sexo del ternero y como covariable la edad de la madre al parto. Las heredabilidades para PN fueron de $\mathrm{h}^{2}{ }_{\mathrm{d}}=0.31, \mathrm{~h}^{2} \mathrm{~m}=0.03 \mathrm{y}$ $\sigma^{2} \mathrm{c}=0.07$. los valores de heredabilidad para PAJ240 fueron moderados y bajos $\left(\mathrm{h}^{2}{ }_{\mathrm{d}}=0.18\right.$, $\mathrm{h}_{\mathrm{m}}^{2}=0.05, \sigma^{2} \mathrm{c}=0.04$ ) en tanto que para PAJ480 fueron de $\mathrm{h}_{\mathrm{d}}{ }_{\mathrm{d}}=0.17, \mathrm{~h}_{\mathrm{m}}{ }_{\mathrm{m}}=0.04, \sigma_{\mathrm{c}}{ }_{\mathrm{c}}=0.06$, sin presentar valores significativos, y la proporción de las varianzas presentaron valores negativos y decreciente en la recta. Las tendencias genéticas fueron creciente y decreciente, indicando que la varianza ambiental afecta negativamente el componente materno y del ambiente permanente para el peso a los 240 y 480 días respectivamente.
\end{abstract}

Palabras clave: Blanco Orejinegro, heredabilidad, efectos genéticos, parámetros productivos

\footnotetext{
${ }^{1}$ Corporación Colombiana de Investigación Agropecuaria - AGROSAVIA, Centro de Investigación Turipaná, Córdoba, Colombia

${ }^{2}$ Corporación Colombiana de Investigación Agropecuaria - AGROSAVIA, Centro de Investigación Tibaitatá, Cundinamarca, Colombia

${ }^{3}$ E-mail: nherrera@agrosavia.co
}

Recibido: 14 de diciembre de 2020

Aceptado para publicación: 21 de agosto de 2021

Publicado: 27 de octubre de 2021

CLos autores. Este artículo es publicado por la Rev Inv Vet Perú de la Facultad de Medicina Veterinaria, Universidad Nacional Mayor de San Marcos. Este es un artículo de acceso abierto, distribuido bajo los términos de la licencia Creative Commons Atribución 4.0 Internacional (CC BY 4.0) [https:// creativecommons.org/licenses/by/4.0/deed.es] que permite el uso, distribución y reproducción en cualquier medio, siempre que la obra original sea debidamente citada de su fuente original 
The aim of this study was to estimate the values of direct and maternal heritability and the variances due to the permanent environment from 1990 to 2012 in the Colombian Creole Blanco Orejinegro breed. In total, 10698 data on growth variables were analyzed for birth weight (BW), adjusted weight at 240 days (PAJ240) and adjusted weight at 480 days (PAJ480). The derivative-free restricted maximum likelihood methodology (DFREML) was used, adjusting an animal model that included direct genetic effects, maternal and variations due to the permanent environment, assuming as fixed effects the year, time of birth, the sex of the calf and as covariate the mother's age at delivery. The heritability for BW were $h^{2} \mathrm{~d}=0.31, \mathrm{~h}^{2} \mathrm{~m}=0.03$ and $\sigma^{2} \mathrm{c}=0.07$. the heritability values for PAJ240 were moderate and low $\left(\mathrm{h}^{2} \mathrm{~d}=0.18, \mathrm{~h}^{2} \mathrm{~m}=0.05, \sigma^{2} \mathrm{c}=0.04\right)$ while for PAJ480 they were $\mathrm{h}^{2} \mathrm{~d}=0.17$, $h^{2} \mathrm{~m}=0.04, \sigma^{2} \mathrm{c}=0.06$, without presenting significant values and the proportion of the variances presented negative and decreasing values on the line. The genetic trends were increasing and decreasing, indicating that the environmental variance negatively affects the maternal component and the permanent environment for the weight at 240 and 480 days, respectively.

Key words: Blanco Orejinegro, heritability, genetic effects, productive parameters

\section{INTRODUCCIÓN}

Uno de los posibles factores que afectan el rendimiento productivo de la ganadería bovina colombiana es el manejo del recurso genético animal, donde no se ha prestado la debida atención a las razas locales (Falconer y Mackay, 1996). Los programas de conservación y de selección deben considerar las posibles variaciones de las características fenotípicas y genotípicas del ganado a través del tiempo, especialmente en poblaciones cerradas donde la homocigosis tiende a ser mayor y las heredabilidades en su gran proporción son bajas, siendo las diferencias fenotípicas entre individuos mayormente debidas a los efectos ambientales (Albuquerque y Meyer, 2004). La estimación de la heredabilidad de los caracteres permite monitorear la variabilidad genética de la población (Falconer y Mackay, 1996).

Las razas bovinas criollas en ambientes tropicales han sobrevivido y evolucionado en condiciones adversas, siendo probable la presencia de animales portadores de genes con características de adaptación en distintas frecuencias si se les compara con razas exóticas, de allí la importancia de caracterizar y utilizar de manera sostenible estos recursos genéticos (Núñez et al., 2016). El objetivo de este estudio, por lo tanto, fue estimar los valores de heredabilidad directa, materna y la variación debida al ambiente permanente de caracteres de crecimiento, así como sus variaciones entre 1990 y 2012 en la raza criolla colombiana Blanco Orejinegro.

\section{Materiales y Métodos}

Los registros productivos para el cálculo de las tendencias de heredabilidades fueron obtenidos de la base de datos del banco de germoplasma de la raza bovina criolla Blanco Orejinegro, utilizando información correspondiente a los años 1990 a 2012. El núcleo de animales forma parte del Centro de Investigación El Nus, de la Corporación Colombiana de Investigación Agropecuaria 
(AGROSAVIA), el cual se encuentra ubicado en las estribaciones de la cordillera central en el municipio de San Roque Antioquia, Colombia. La zona presenta altitudes entre 800 y $1300 \mathrm{msnm}$, temperatura media anual de $23{ }^{\circ} \mathrm{C}$, humedad relativa de $87 \%$ y precipitación bimodal anual de $2200 \mathrm{~mm}$.

El núcleo en conservación se maneja bajo un sistema de apareamiento circular cíclico para mantener un índice bajo de consanguinidad, conformando grupos por familias en función a su parentesco. Se asumió como efectos fijos el año, época de nacimiento y sexo, y como covariable la edad de la vaca al parto. Se analizaron 10698 animales con registros productivos e información completa, para peso al nacimiento $(\mathrm{n}=4267)$, peso ajustado al destete $(240 \mathrm{~d}$; $\mathrm{n}=3299)$ y 16 meses ( $480 \mathrm{~d} ; \mathrm{n}=3132$ ) (Cuadro 1).

Se determinaron las heredabilidades directas, las maternas y las varianzas debidas al ambiente permanente entre 1990 y 2012 . Se graficaron las tendencias en el tiempo de estas variables incluyendo las respectivas ecuaciones de regresión y coeficientes de determinación. Los componentes de varianza y covarianza empleadas para estimar los valores de heredabilidad fueron obtenidos a través de un análisis unicarácter empleando el método de máxima verosimilitud restricta aplicada a un modelo animal, mediante el programa DFREML.

Se utilizó el modelo mixto descrito por Henderson (1975): $Y=X \beta+Z a+Z m+Z c$ $+e$, donde $Y$ : vector de observaciones, $\beta$ : vector de soluciones para efectos fijos (año de nacimiento, sexo, edad de la vaca al parto), $X$ : matriz de incidencia de los efectos fijos, $Z$ : matriz de incidencia de los efectos aleatorios, $a$ : vector de soluciones para valores genéticos, $m$ : vector de soluciones para el efecto materno, $c$ : vector de soluciones para efectos del ambiente permanente, y $e$ : valores residuales.
La estructura de (co)varianza de los efectos aleatorios para las características de crecimiento evaluadas se resume en la siguiente expresión:

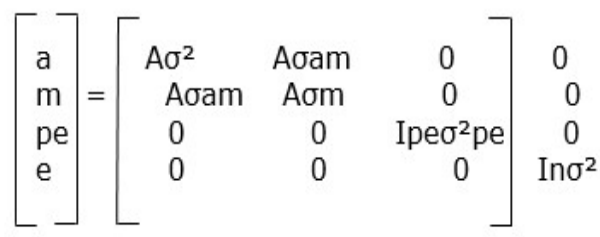

Los cálculos de heredabilidad aditiva directa fueron hechos mediante componentes de varianza a partir del modelo animal, utilizando la siguiente fórmula: $\mathrm{h}_{\mathrm{d}}{ }_{\mathrm{d}}=\left(\sigma_{\mathrm{a}}^{2}\right) /$ $\left(\sigma_{\mathrm{f}}^{2}\right)$, donde $h_{d}^{2}$ : heredabilidad aditiva directa, $\sigma_{a}^{2}$ : varianza genética aditiva directa, $\sigma_{f}^{2}$ : varianza fenotípica total.

La heredabilidad para los componentes genéticos de tipo materno se estimó utilizando la siguiente fórmula: $\mathrm{h}_{\mathrm{m}}^{2}=\left(\sigma_{\mathrm{m}}^{2}\right) /\left(\sigma_{\mathrm{f}}^{2}\right)$, donde $h^{2}$ : heredabilidad de los componentes genéticos maternos, $\sigma_{m}^{2}$ : varianza genética materna, $\sigma_{f}^{2}$ : varianza fenotípica total.

La estimación del índice de repetibilidad $(t)$ de los pesos al nacimiento (PN), al destete (P240) y a los 480 días (P480) para las madres que tenían registros repetidos se halló mediante componentes de varianza maternos: $t$ $=\sigma_{\mathrm{a}}^{2}+\sigma_{\mathrm{c}}^{2} /\left(\sigma_{\mathrm{f}}^{2}\right)$, donde $\sigma_{a}^{2}$ : varianza genética aditiva directa, $\sigma_{c}^{2}$ : varianza del ambiente permanente, $\sigma_{\mathrm{f}}^{2}$ : varianza fenotípica total.

\section{Resultados}

\section{Peso al Nacimiento}

La heredabilidad del peso al nacimiento para los 23 años del estudio (1990-2012) fue $\mathrm{de}^{2}{ }_{\mathrm{d}}=0.31$, el cual se considera alta. Asimismo, la heredabilidad materna y la varianza debidas al ambiente permanente mostraron valores promedios bajos $\left(\mathrm{h}_{\mathrm{m}}^{2}=0.03 \mathrm{y}^{2}{ }_{\mathrm{c}}=\right.$ 0.07 , respectivamente). 


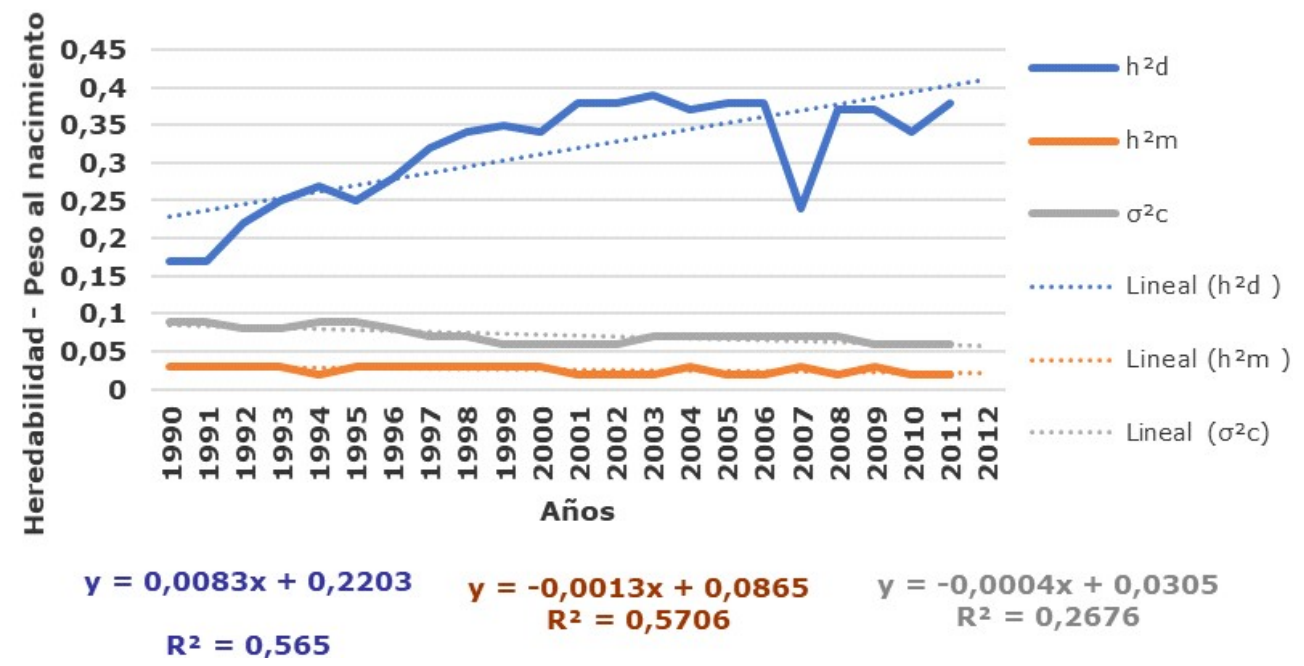

Figura 1. Tendencias de heredabilidad directa $\left(\mathrm{h}^{2}{ }_{\mathrm{d}}\right)$, materna $\left(\mathrm{h}_{\mathrm{m}}^{2}\right)$ y la varianza debidas al ambiente permanente $\left(\sigma_{c}^{2}\right)$ para peso al nacimiento en bovinos Blanco Orejinegro, Colombia

El menor valor de heredabilidad directa se observó en $1990\left(\mathrm{~h}_{\mathrm{d}}{ }_{\mathrm{d}}=0.14\right)$ y la mayor en $2004\left(\mathrm{~h}_{\mathrm{d}}^{2}=0.39\right)$, lo cual corresponde a un aumento del $64 \%$ en este periodo, posiblemente debido a la disminución de los efectos ambientales que intervienen en este carácter.

La heredabilidad directa a través de los años mostró una tendencia creciente para el peso al nacer, pero además se observa una dispersión de su variabilidad. La ecuación de regresión indica un crecimiento promedio anual de $0.83 \%$ del valor de heredabilidad directa $\left(\mathrm{R}^{2}=0.56\right)$. La población de estudio busca mantener la variabilidad genética y de esta forma se espera una tendencia a aumentar la heterocigosis y que las frecuencias génicas se encuentren con valores intermedios.

La heredabilidad materna presentó el mayor valor en $1990\left(\mathrm{~h}_{\mathrm{m}}^{2}=0.04\right)$ y el menor en $2009\left(\mathrm{~h}_{\mathrm{m}}^{2}=0.02\right)$, con una tendencia decreciente anual de $-0.13 \%$. El comportamiento del coeficiente de determinación de la línea recta fue de $\mathrm{R}^{2}=0.57$. lo cual indica una dispersión media de los puntos sobre la recta de regresión, manteniendo una baja heredabilidad en el tiempo.

La varianza del ambiente permanente a través de los años presentó un valor promedio de $\sigma^{2}{ }_{\mathrm{c}}=0.07$, considerado como un valor bajo, teniendo en cuenta la tendencia de la proporción de la varianza debida al ambiente permanente (Figura 1). Hubo una disminución leve del valor promedio anual indicado por el coeficiente de regresión negativo $\left(B_{l}=-\right.$ 0.0004 ) tomando un valor inicial de 0.09 en 1990 , pero con bajo ajuste en la curva de regresión $\left(\mathrm{R}^{2}=0.26\right)$.

\section{Peso al Destete Ajustado a 240 Días}

Se observa un valor promedio moderado de la heredabilidad directa de $\mathrm{h}^{2}=0.18, \mathrm{y}$ una heredabilidad materna de $\mathrm{h}_{\mathrm{m}}^{2}=0.05$, considerada baja. Este valor indica que el componente genético aditivo relacionado con la habilidad materna de la madre tiene un efecto bajo sobre la variabilidad del carácter y que su variación está influenciada en mayor medida por factores ambientales no controlados. 


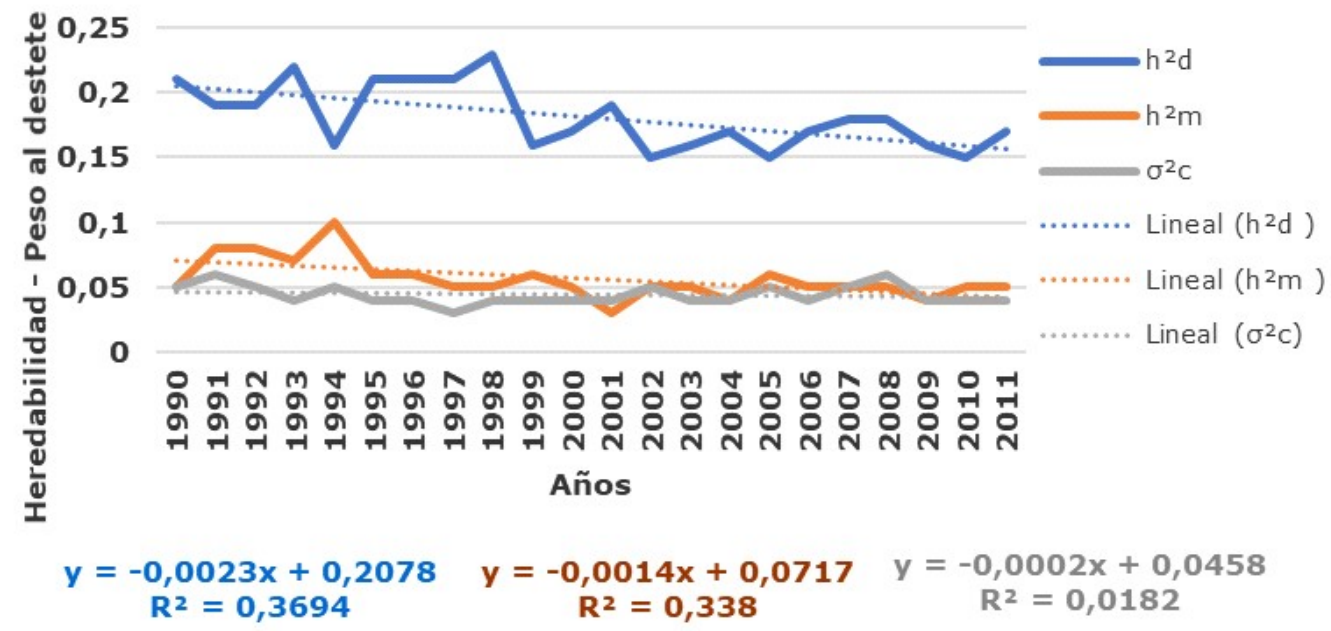

Figura 2. Tendencias de heredabilidad directa $\left(\mathrm{h}_{\mathrm{d}}^{2}\right)$, materna $\left(\mathrm{h}_{\mathrm{m}}^{2}\right)$ y varianza debidas al ambiente permanente $\left(\sigma_{c}^{2}\right)$, para peso ajustado a los 240 días en bovinos Blanco Orejinegro, Colombia

La Figura 2 muestra los valores de heredabilidad directa, materna y las varianzas debidas al ambiente permanente en el peso ajustado a 240 días. En 1998 se obtuvo el mayor valor $\left(\mathrm{h}^{2}{ }_{\mathrm{d}}=0.23\right)$, mostrando posteriormente un comportamiento decreciente $\left(B_{1}\right.$ $=-0.00023)$ y variable, con el menor valor $\left(\mathrm{h}_{\mathrm{d}}^{2}=0.15\right)$ para los años 2002, 2005 y 2015, el cual se considera un valor moderado. Estas variaciones anuales podrían deberse al cambio en los efectos ambientales temporales en la población y la forma en que se evalúa el fenotipo, o por mayores variaciones en la edad al destete en los últimos años, lo que implica que el ajuste lineal a 240 días pueda tener un bajo coeficiente de determinación.

Los efectos aditivos maternos obtuvieron un valor promedio de $\mathrm{h}_{\mathrm{m}}^{2}=0.06$ con un comportamiento decreciente en la recta $\left(B_{1}\right.$ $=-0.0014)$ y un coeficiente de determinación bajo $\left(\mathrm{R}^{2}=0.33\right)$. Se debe considerar las influencias ambientales y efectos maternos temporales que afectan este carácter, los cuales pueden tener un mayor efecto sobre la variación del peso que el componente materno.
La varianza del ambiente permanente presentó un valor promedio bajo $\left(\mathrm{h}^{2}=0.04\right)$. Esta variación se produce entre los partos sucesivos de un mismo animal sobre su progenie para las características productivas; sin embargo, el carácter mostró un valor bajo y constante a través de los años, con un valor inicial de $\sigma^{2}{ }_{\mathrm{c}}=0.04$ en 1990, un comportamiento decreciente $\left(B_{I}=-0.0002\right)$ como lo indica la recta, y un coeficiente de determinación bajo $\left(\mathrm{R}^{2}=0.018\right)$, probablemente debido a que los valores de las tendencia están relacionados con secuelas de enfermedades de la madre que afecten la producción de leche.

\section{Peso Ajustado a los 480 Días}

La heredabilidad directa tuvo un valor promedio moderado de $\mathrm{h}_{\mathrm{d}}{ }_{\mathrm{d}}=0.17$, en tanto que la heredabilidad materna y la varianza debidas al ambiente permanente mostraron valores bajos $\left(\mathrm{h}_{\mathrm{m}}^{2}=0.04 \mathrm{y} \sigma_{\mathrm{c}}^{2}=0.06\right.$, respectivamente). 


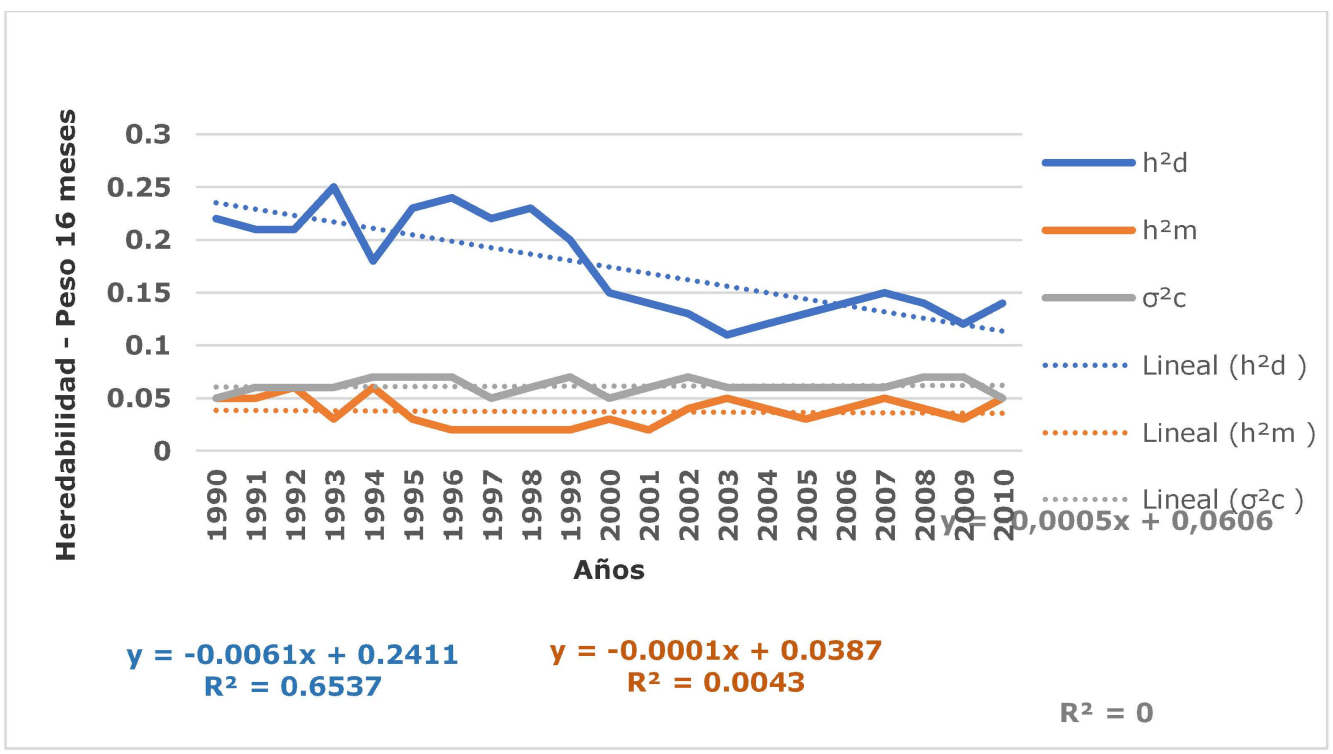

Figura 3. Tendencias de heredabilidad directa $\left(\mathrm{h}^{2}{ }_{\mathrm{d}}\right)$, materna $\left(\mathrm{h}^{2}{ }_{\mathrm{m}}\right)$ y debidas al ambiente permanente $\left(\sigma_{c}^{2}\right)$, para peso ajustado a los 480 días, en bovinos Blanco Orejinegro, Colombia

En la Figura 3 se observa que en 1993 se presentó el mayor valor de heredabilidad directa $\left(\mathrm{h}^{2}{ }_{\mathrm{d}}=0.25\right)$ y la menor en $2003\left(\mathrm{~h}^{2}{ }_{\mathrm{d}}=\right.$ 0.11 ), disminución probablemente relacionada directamente por efecto del ambiente. Los valores de heredabilidad directa indican un comportamiento decreciente en la recta $\left(B_{I}=\right.$ - 0.006) en el tiempo. La expresión materna aditiva mínima tuvo valores de $\mathrm{h}_{\mathrm{m}}{ }_{\mathrm{m}}=0.05$, considerados como bajos y la tendencia muestra en la recta un decrecimiento $\left(B_{1}\right.$ $=-0.0001)$.

La proporción de la varianza debidas al ambiente permanente fue de $\sigma^{2}{ }_{c}=0.06$, lo cual indica la poca influencia de las diferencias entre partos sucesivos de las madres en la manifestación genética del parámetro. La proporción de la varianza debida a efectos de ambiente permanente en la recta presentó un valor bajo decreciente $\left(B_{l}=-0.0005\right)$, con valores variables entre 1990 y 2000 , pero entre 2000 hasta 2004 con variaciones más estables.

\section{Discusión}

\section{Peso al Nacimiento}

La heredabilidad directa fue superior a los valores de heredabilidad directa de $\mathrm{h}_{\mathrm{d}}{ }_{\mathrm{d}}=$ 0.19 en la raza Romosinuano en México (Martínez et al., 2018), al igual que en Colombia $\left(\mathrm{h}_{\mathrm{d}}{ }_{\mathrm{d}}=0.19\right)$, además de valores bajos para heredabilidad materna $\left(\mathrm{h}_{\mathrm{m}}^{2}=0.03\right)$ (Ossa et al., 2014), pero estos últimos autores reportaron valores superiores y altos para la heredabilidad directa $\left(\mathrm{h}_{\mathrm{d}}{ }_{\mathrm{d}}=0.42\right)$ para el Blanco Orejinegro. Bejarano (2016), de otra parte, en la raza Romosinuano reportó valores moderados $\left(h^{2}{ }_{d}=0.25\right)$, en tanto que Ndofor et al. (2012) reportaron valores altos de heredabilidad directa $\left(\mathrm{h}^{2}{ }_{\mathrm{d}}=0.39\right)$ y valores bajos para la heredabilidad materna $\left(\mathrm{h}_{\mathrm{m}}{ }_{\mathrm{m}}=\right.$ 0.05) en bovinos Gudali en África, siendo estos valores cercanos a los encontrados en este estudio. 
En Austria, en la raza Murboden se estimaron valores inferiores de heredabilidades directas y maternas $\left(\mathrm{h}_{\mathrm{d}}^{2}=0.17 \mathrm{y} \mathrm{h}_{\mathrm{m}}{ }_{\mathrm{m}}=0.07\right.$, respectivamente (Eaglen et al., 2013), así como en ganado Fogera con valores bajos de heredabilidad directa, materna y varianza del ambiente permanente $\left(\mathrm{h}_{\mathrm{d}}{ }_{\mathrm{d}}=0.06, \mathrm{~h}_{\mathrm{m}}{ }_{\mathrm{m}}=0.03 \mathrm{y}\right.$ $\sigma^{2}{ }_{c}=0.05$, respectivamente) utilizando tres modelos de análisis (Bekele et al., 2018). Otros resultados muestran valores altos y similares de heredabilidad directa $\left(\mathrm{h}_{\mathrm{d}}{ }_{\mathrm{d}}=0.34\right)$ en la raza Borano (Wasike et al., 2009) en África y valores moderado de heredabilidad directa $\left(\mathrm{h}^{2}{ }_{\mathrm{d}}=0.21\right)$ en ganado Brown Swiss en México, además de un bajo valor de heredabilidad materna $\left(\mathrm{h}_{\mathrm{m}}^{2}=0.05\right)$ (Segura et al., 2012). Asimismo, otros estudios reportaron valores moderados y bajos de heredabilidades $\left(\mathrm{h}_{\mathrm{d}}^{2}=0.18, \mathrm{~h}_{\mathrm{m}}{ }_{\mathrm{m}}=0.006, \sigma_{\mathrm{c}}^{2}=\right.$ $0.05)$ en la raza criolla Costeño con Cuernos (Vergara et al., 2018), y de $\mathrm{h}^{2}=0.25$ en la raza Nellore (Chud et al., 2014).

Los resultados de este estudio muestran que los valores encontrados están dentro de los valores reportados en diferentes razas y continentes. Además, se evidencia una variabilidad genética para el rasgo, lo cual indica la necesidad de realizar la evaluación de este parámetro para monitorear los resultados del programa de conservación. Este parámetro podría utilizarse para la selección de animales que poseen bajo peso al nacimiento, lo que facilita el parto por ser una característica que manifiestan las razas criollas colombianas.

\section{Peso Ajustado a los 240 Días}

En la raza Romosinuano en México se obtuvo un valor moderado y bajo para la heredabilidad directa $\left(\mathrm{h}_{\mathrm{d}}^{2}=0.21\right)$, así como una baja heredabilidad materna $\left(\mathrm{h}_{\mathrm{m}}{ }_{\mathrm{m}}=0.04\right)$ (Martínez et al., 2018). De otra parte, Ossa et al. (2014) reportaron para dicha raza valores inferiores de heredabilidad directa y materna $\left(\mathrm{h}_{\mathrm{d}}^{2}=0.13 \mathrm{y} \mathrm{h}_{\mathrm{m}}{ }_{\mathrm{m}}=0.08\right.$, respectivamente). Asimismo, Ndofor-Foleng et al. (2012) reportaron valores moderados y superiores para las heredabilidades directa $y$ materna $\left(\mathrm{h}_{\mathrm{d}}{ }_{\mathrm{d}}=0.25 \mathrm{y} \mathrm{h}_{\mathrm{m}}^{2}=0.11\right.$, respectivamente) para la raza Gudal, en tanto que Eaglen et al. (2013) para la raza Murboden estimaron valores superiores y altos para la heredabilidad directas $\left(\mathrm{h}_{\mathrm{d}}{ }_{\mathrm{d}}=0.49\right)$ y valores moderados pero superiores para heredabilidad materna $\left(\mathrm{h}_{\mathrm{m}}^{2}=0.11\right)$.

En animales Simmental se han reportado valores moderados de $\mathrm{h}_{\mathrm{d}}{ }_{\mathrm{d}}=0.25$ (Amaya et al., 2020), pero superiores a los encontrados en este estudio, en tanto que en Cuba se reportaron valores altos $\left(\mathrm{h}^{2}{ }_{\mathrm{d}}=0.45\right)$ para ganado cebú, aunque valores similares para la heredabilidad materna $\left(\mathrm{h}^{2}=0.21\right)$ (Palacios et al., 2019). De otro lado, Wasike et al. (2009), para la raza Borano en África reportaron valores inferiores de heredabilidad directa $\left(\mathrm{h}_{\mathrm{d}}^{2}=0.12\right)$ y moderada, pero bajos para la heredabilidad materna $\left(\mathrm{h}^{2} \mathrm{~m}=0.14\right)$, encontrando que el efecto del ambiente materno contribuye en un 16,4 y $10 \%$ de la varianza fenotípica total para los pesos al destete, $12 \mathrm{y}$ 18 meses, respectivamente. Si bien el componente materno para incrementar los pesos por selección en relación con la habilidad materna no se considera recomendable para este tipo de rebaños, la mejora de las condiciones ambientales en las vacas en los periodos de lactación es oportuna.

En la raza criolla Costeño con Cuernos se han reportado valores altos de heredabilidad directa $\left(\mathrm{h}^{2}=0.33\right)$ y valor moderado para la heredabilidad materna $\left(\mathrm{h}^{2}{ }_{\mathrm{m}}=\right.$ $0.10)$, pero baja varianza debida al ambiente permanente $\left(\sigma^{2}{ }_{c}=0.02\right)$, donde los valores de heredabilidad materna y varianza del ambiente permanente fueron inferiores a los del presente estudio (Vergara et al., 2018). En forma similar, se han reportado valores moderados y bajos de heredabilidades $\left(\mathrm{h}_{\mathrm{d}}{ }_{\mathrm{d}}=0.28\right)$ en Nellore (Chud et al., 2014) y en cebú rojo $\left(\mathrm{h}_{\mathrm{d}}{ }_{\mathrm{d}}=0.11, \mathrm{~h}_{\mathrm{m}}^{2}=0.03, \sigma_{\mathrm{c}}^{2}=0.10\right)$ (Guillén et al., 2012).

Los resultados muestran que hay un amplio rango de valores para los parámetros del carácter peso al destete, quizás como consecuencias de las diferencias en la raza, 
época, modelos de análisis y condiciones de crianzas entre otras. Estos resultados además indican que existe variabilidad en el carácter la cual puede ser utilizada para desarrollar programas de selección.

\section{Peso Ajustado a 480 Días}

En Colombia se han reportado valores medios y similares para la heredabilidad directa en la raza Romosinuano de $\mathrm{h}_{\mathrm{d}}{ }_{\mathrm{d}}=0.21$ (Ossa et al., 2014), así como heredabilidades altas $\left(\mathrm{h}^{2}{ }_{\mathrm{d}}=0.38\right)$ en el criollo Blanco Orejinegro y de $\mathrm{h}_{\mathrm{d}}=0.37$ en Romosinuano (Bejarano, 2016). Asimismo, para la raza Gudali se reportaron valores inferiores de heredabilidad materna $\left(\mathrm{h}_{\mathrm{m}}^{2}=0.07\right)$ (NdoforFoleng et al., 2012). Por otro lado, Wasike et al. (2009), con animales Borano en Kenia, reportaron estimaciones de heredabilidad directa de $\mathrm{h}_{\mathrm{d}}{ }_{\mathrm{d}}=0.08$ para características de crecimiento a los 18 meses de edad y de $\mathrm{h}^{2}{ }_{\mathrm{d}}$ $=0.14$ a los 24 meses, siendo estos valores inferiores a los reportados en este trabajo.

Se reportan valores altos de heredabilidad directa $\left(\mathrm{h}_{\mathrm{d}}{ }_{\mathrm{d}}=0.36\right)$ en ganado Costeño con Cuernos (Vergara et al., 2018), así como valores superiores de $\mathrm{h}_{\mathrm{d}}^{2}=0.28$, pero bajos de $\sigma_{c}^{2}=0.03$ para la varianza debida al ambiente permanente ganado cebú rojo (Guillén et al., 2012).

Es importante indicar que la heredabilidad no es solo una propiedad del carácter sino también de la población, así como de las condiciones ambientales en las que los individuos se desarrollan y de la forma en que se evalúa el fenotipo; por lo tanto, el valor depende de la forma en que afecte cada uno de estos factores, que en sistemas de producción extensiva en el trópico, presentan un efecto considerable y de magnitud superior que en sistemas intensivos donde se puede tener una mayor precisión en la estimación del valor fenotípico para un carácter, debido a las condiciones ambientales más uniformes .
La varianza aditiva directa o materna está en función de las frecuencias génicas en una población y puede cambiar por efectos de su manejo, encontrándose que en poblaciones pequeñas con censo reducido o donde se ha dado tasas considerables de consanguinidad, la heredabilidad puede verse reducida drásticamente (Falconer y Mackay, 1996).

\section{Conclusiones}

Las evaluaciones genéticas para características de crecimiento en la raza criolla Blanco Orejinegro en una población en conservación muestran que:

- La heredabilidad directa presenta valores altos para el peso al nacimiento y valores moderados para los pesos ajustados a los 240 y 480 días,

- La heredabilidad materna y la varianza al ambiente permanente presentan valores bajos para los tres caracteres de crecimiento.

- El manejo o ausencia de selección en esta población en conservación evidencia que la varianza ambiental afecta negativamente el componente materno y del ambiente permanente.

- Para mejorar los pesos al destete y los pesos a 16 meses se requiere tomar medidas sobre las fuentes de variación significativas.

\section{Agradecimientos}

A la Corporación Colombiana de Investigación Agropecuaria (AGROSAVIA) y MADR por la financiación de los recursos para el desarrollo del proyecto de conservación de la colección biológica de la raza bovina Blanco Orejinegro del banco de germoplasma animal del C.I El Nus. 


\section{Literatura Citada}

1. Albuquerque LG, Meyer K. 2004. Estimates of covariance functions for growth of Nelore cattle applying a parametric correlation structure to model within-animal correlations. Livest Prod Sci 93: 213-222. doi: 10.1016/j.livprodsci.2004.10.006

2. Amaya MA, Martínez SR, CerónMuñoz M. 2020. Parámetros genéticos para crecimiento y reproducción en ganado Simmental mediante parentesco por pedigrí y genómico. Rev MVZ Córdoba 25: e1520. doi: 10.21897/rmvz.1520

3. Bejarano D. 2016. Estudio de asociación genómica para características de crecimiento en las razas bovinas criollas Blanco Orejinegro y Romosinuano. Tesis de Maestría. Bogotá, Colombia: Univ. Nacional de Colombia. 175 p.

4. Bekele A, Wuletaw Z, Haile A, Gizaw $S$, Mekuriaw G 2018. Genetic para-meter estimation of growth traits of Fogera cattle. Glob J Anim Breed Genet 6: 21-33.

5. Chud TCS, Caetano SL, Buzanskas $M E$, Grossi DA, Guidolin DGF, Nascimento GB, Rosa JO, et al. 2014. Genetic analysis for gestation length, birth weight, weaning weight, and accumulated productivity in Nellore beef cattle. Livest Sci 170: 16-21. doi: 10.1016/ j.livsci.2014.09.024

6. Eaglen SAE, Fuerst-Walt B, Fuerst C, Sölkner J. 2013. Evaluating maternal traits in the Austrian Murboden cattle: genetic parameters and inbreeding depression. Interbull Bull 47: 230-235.

7. Falconer DS, Mackay TFC. 1996. Introducción a la genética cuantitativa. Ed Acribia. $500 \mathrm{p}$.

8. Guillén Trujillo A, Guerra Iglesias D, Ávila Serranoc N, Palacios Espinosa $A$, Ortega Pérez $R$, Espinoza Villavicencio J. 2012. Parameters and genetic trends for weight at weaning and 18 months of age in red zebu cattle in Cuba. Rev Mex Cienc Pecu 3: 19-31.
9. Henderson CR. 1975. Best linear unbiased prediction under a selection model. Biometrics 31: 423-447.

10. Martínez Rocha RE, Ramírez Valverde R, Núñez Domínguez R, García Muñiz JG. 2018. Parámetros y tendencias genéticas de variables de crecimiento para bovinos Romosinuano en México. Nova Scientia 10: 310-325. doi: 10.21640/ ns.v10i21.1595

11. Ndofor-Foleng HM, Ebangi AL, Agu CI, Okenyi N. 2012. Estimation of genetic parameters for preweaning and postweaning growth traits in the Gudali beef cattle using multiple trait derivative free restricted maximum likelihood. Afr J Biotechnol 11: 14410-14416. doi: 10.5897/AJB12.1038

12. Núñez Domínguez R, Ramírez Valverde R, Saavedra Jiménez LA, García Muñiz JG. 2016. La adaptabilidad de los recursos zoogenéticos criollos, base para enfrentar los desafíos de la producción animal. Arch Zootec 65: 461-468.

13. Ossa Saraz GA, Narváez Pérez HJ, Noriega Márquez JG, Pérez Garcia JE, Vergara Garay OD. 2014. Parámetros y tendencias genéticas para características de crecimiento en una población de ganado criollo Romosinuano. Livestock Res Rural Develop 26(10). [Internet]. Disponible en: http:// www.lrrd.org/lrrd26/10/ossa26191.html

14. Palacios Espinosa A, EspinozaVillavicencio JL, Menéndez-Buxadera A. 2019. Parámetros genéticos para peso al destete y rasgos reproductivos en ganado cebú de Cuba. Nova Scientia 11: 1-25. doi: 10.21640/ns.v11i22.1672

15. Segura-Correa JC, Chin-Colli RC, Magaña-Monforte JG, NúñezDomínguez $R$. 2012. Genetic parameters for birth weight, weaning weight and age at first calving in Brown Swiss cattle in Mexico. Trop Anim Health Pro 44: 337-341. doi: 10.1007/s11250011-0026-8 
16. Vergara Garay O, Pérez García J, Noriega Márquez J, Cencio Martínez, Martínez Humanez N. 2018. Análisis multicarácter para estimar componentes de (co)varianza en características de crecimiento en bovinos costeño con cuernos. Livestock Res Rural Develop 30(9). [Internet]. Disponible en: http:// www.lrrd.org/lrrd30/9/overg30161.html
17. Wasike CB, Indetie D, Ojango JM, Kahi AK. 2009. Direct and maternal (co)variance components and genetic parameters for growth and reproductive traits in the Boran cattle in Kenya. Trop Anim Health Pro 41: 741-748. doi: 10.1007/s11250-008-9246-y 